\title{
The Journal of Adhesion
}

\section{Mounting of Accelerometers with Structural Adhesives: Experimental Characterization of the Dynamic Response}

\section{Marco Cocconcelli \& Andrea Spaggiari}

To cite this article: Marco Cocconcelli \& Andrea Spaggiari (2015): Mounting of Accelerometers with Structural Adhesives: Experimental Characterization of the Dynamic Response, The Journal of Adhesion, DOI: 10.1080/00218464.2015.1120197

To link to this article: http://dx.doi.org/10.1080/00218464.2015.1120197

Accepted author version posted online: 15 Dec 2015.

Submit your article to this journal $\sqsubset$

Џlll Article views: 22

View related articles

View Crossmark data $\nearrow$ 


\title{
MOUNTING OF ACCELEROMETERS WITH STRUCTURAL ADHESIVES: EXPERIMENTAL CHARACTERIZATION OF THE DYNAMIC RESPONSE
}

\section{Marco Cocconcelli}

Department of Engineering Sciences and Methods, University of Modena and Reggio Emilia, Italy E-mail: marco.cocconcelli@unimore.it

\author{
Andrea Spaggiari \\ Department of Engineering Sciences and Methods, University of Modena and Reggio Emilia, \\ Italy
}

E-mail: andrea.spaggiari@unimore.it (corresponding author)

\begin{abstract}
.
The use of accelerometers to monitor the vibrations of either complex machinery or simple components involves some considerations about the mounting of the sensor to the structure. Different types of mounting solutions are commonly used, but in all cases they can be classified in one of these categories: stud mounting, screw mounting, adhesive mounting, magnetic mounting and probe sensing. Indeed each of them has a specific field of application depending on e.g. the mounting surface conditions, the temperature, the accessibility to the specific mounting point, etc. The choice of the mounting solution has an important effect on the accuracy of the usable frequency response of the accelerometer, since the higher the stiffness of the fixing, the higher the low-pass frequency limit of the mounting. This paper specifically focuses on
\end{abstract}


adhesive mounting of accelerometers, which includes a great number of different products from the temporary adhesives like the beeswax to the permanent ones like cyanoacrylate polymers. Among the variety of commercial adhesives, three specific products have been experimentally compared to assess their transmissivity and the results are reported in this paper. A two component methylmethacrylate (HBM X60), a modified silane (Terostat 737) and a cyanoacrylate (Loctite 454) adhesives have been used to joint two aluminium bases, one connected to an accelerometer and the other to the head of electromagnetic shaker. A design of experiments (DOE) approach was used to test the system at several levels of amplitude and frequency of the external sinusoidal excitation supplied by the shaker.

Keywords: mechanical properties of adhesives, cyanoacrylate, silicones , accelerometers mounting, vibration transmissivity, statistical analysis

\section{INTRODUCTION}

The vibration monitoring activity is the most used technique to assess the working condition of a machinery. The aims of this activity are: monitoring the noise level of a component, monitoring the vibration transmitted to close system components, increasing the precision of an end-effector, reducing the health risk for a user, and determining possible mechanical damage of specific components like bearing and gears. Regardless the specific aim, all the vibration measurements are normally performed by means of a piezoelectric accelerometer or based on Micro ElectroMechanical Systems (MEMS) technology. The development of both piezoelectric and MEMS technology leads to robust products, resistant to hostile environments, with small dimensions and 
a wide frequency response. Since the introduction of the first piezoelectric accelerometer was in the '50s (MEMS come lately), a relevant amount of papers on signal processing can be traced in literature, defining the know-how on vibration analysis so far. It is interesting to observe that despite the thousands of papers describing how to treat the vibration signal, only few papers focuses on a correct setup of the vibration sensors [1]. This practical aspect is demanded to the university courses on vibrations analysis, personal experience or to information given by the accelerometer suppliers [2-4]. This paper focuses on a specific aspects of the accelerometer setup, the mounting between the sensor and the surface of the component. The most frequent solutions adopted can be classified in few categories: stud mounting, screw mounting, adhesive mounting, magnetic mounting and probe mounting. Each of them has a specific field of application depending on e.g. the mounting surface conditions, the temperature, the accessibility to the specific mounting point, etc. A detailed description of all the mounting techniques is not the purpose of the paper, but in the classic handbook on shock and vibration [5] all details are provided. Among the several techniques, the stud/screw and adhesive mounting are the most used. These types of mounting results in a rigid connection with high stiffness and wide frequency range response. The higher the stiffness of the fixture, the higher the low-pass frequency limit of the mounting. While an high stiffness is always provided through the screw coupling, the stiffness of the adhesive bond depends on the physical characteristics of the adhesive, which are not always supplied by the vendor. The aim of this paper is to assess experimentally the dynamic response of three different adhesives which cover the different type of structural adhesive used in on-field applications. Structural adhesives are a standard in the 
fastening of sensors and accelerometers, since they provide a fast and simple mounting without the need of drilling permanent threaded holes on the chassis of the machine [5].

The aim of the paper is to assess which is the effect of the adhesive film used to bond the stud in terms of vibration monitoring and signal transmission. Despite the wealth of information about static mechanical properties of adhesive, like elastic modulus and strength, often supplied directly from the manufacturer, technical literature reports less information about the dynamic of adhesives and mainly in case of high strain rate loading [6] and viscoelastic properties [7]. This work investigates the effects of different adhesives on the vibration monitoring commonly used in industry. The adhesives are used to join two aluminium bases, the first one connected with a threaded coupling to an accelerometer and the second one coupled with the head of electromagnetic shaker. The description of the experimental procedure and the detailed experimental set up are shown in Section 2.3. The decision to use an aluminium substrate is one of the most demanding condition for the adhesive, since aluminium surface properties are not ideal for the adhesive bonding due to oxide formation. According to technical literature the more reactive the surface is (such as mild steel or brass) the stronger the bonding will be. The Materials and Method section describes as well the design of experiment approach used. It consist of three variables in the experimental plan: adhesive type, frequency and amplitude of the signal. The adhesive choice is led by a practical consideration: typically the adhesive mounting of an accelerometer is performed with a commercial, general purpose adhesive which can be found in every industrial site. Thus a superglue, a modified silane adhesive and a strain gauge adhesive, often recommended by the accelerometer manufacturer, are considered in the experimental plan. The frequency and the amplitude are chosen in order to span the entire range 
of the electromagnetic shaker used. The section 3.1 show how the post processing of the data is carried out. The vibrator signal is post-processed to extract two significant output parameters: the SAR, which is the amplitude of the power spectrum at the excitation frequency and its weight percent over the overall energy of the signal. These data are elaborated with a statistical software to evaluate which variable affects the system responses and which is the adhesive effect on the vibration monitoring. The Section 3.2 exploits the ANOVA (Analysis of Variance) technique to estimate which are the main effect on the two selected responses by means of half normal plots and variable interactions diagrams. In the conclusions some relevant considerations are carried out and some practical hints for the adhesive bonding of accelerometers are presented.

\section{Materials And Method}

\subsection{Design of Experimental plan}

The concept of Design of Experiments (DoE) was developed to optimize the experimental effort for multiple variables involved in a problem [8]. The same principles can be also applied to numerical studies, treating each numerical analysis with a different set of problem parameters as a 'virtual experiment' [9]. In this work the DoE technique was used to estimate the factors with the strongest influence on the dynamic mechanical response of adhesive for mounting accelerometers. Three factors were considered in the analysis namely:
a) Excitation frequency, $\mathrm{f}$
b) Excitation amplitude, A 
c) Adhesive type, G

Four values of excitation frequency, f, are examined viz. $5 \mathrm{~Hz}, 2500 \mathrm{~Hz}, 5000 \mathrm{~Hz}$ and $7500 \mathrm{~Hz}$, in order to span the entire range of the accelerometer. (Using the DoE notation, $\mathrm{f}$ is varied over four levels). The values chosen for the excitation amplitude are expressed as a percentage of the maximum amplitude provided by the shaker, $A=50 \%$ and $A=100 \%$. The adhesive type are four, G1 is the commercial superglue (Loctite 454), the G2 is an elastic adhesive modified silane (Terostat 937), both produced by Henkel Adhesive, Cerano (NO), Italy. The G3 is a very stiff two component methylmethacrialte adhesive (HBM X60), produced by HBM, Milan, Italy and G4 is a reference configuration in which there is no adhesive but continuum material. We did not added the beeswax as another level, even though it is one of the most typical way to connect an accelerometer for two main reasons. First the beeswax is stiff in axial direction, but compliant along the plane, therefore it is not recommended for triaxial accelerometers. Second, we wanted to test a general connection, able to mount an accelerometer also upside-down, so the beeswax is not applicable.

We decided to pick three very different adhesives also in term of viscoelastic behaviour. A DMA on the selected adhesives would have been useful, but in literature we could not trace precise information about the viscoelastic behaviour of the adhesives chosen. We can only qualitatively assess that the adhesives have very different viscoelastic behaviour based on the chemistry of the polymers involved [10,11]. in particular the stiffer adhesives (G1 and G3) have limited viscous effect, while the softer one (G2) is quite viscoelastic. According to our intent the viscoelasticity could play a potential beneficial role since it stiffen the adhesive layer improving the signal 
transmission, as shown in for similar adhesives. A summary of the variable levels is reported in Table 1 with a schematic of the mounting configuration. The two aluminium blocks (white squares in the schematics of Table 1) are connected with a thin layer of adhesive (in black), the lower one is connected to the shaker, the upper one has a threaded connection for the accelerometer (grey semicircles). The only difference for the reference configuration with respect to the other cases, is the mass of the single block which is double weighted in order to keep the same nominal natural frequency. We decided not to consider the adhesive layer thickness in the experimental plan even though it is important in the adhesive stiffness and strength [12-17], since it is a typical uncontrolled parameter in a practical application of a bonded accelerometer. The description of the deposition of the adhesive layer and the curing process is reported in Section 2.3.

Among the several DoE techniques available, a full factorial plan is adopted, with three replicates for each experimental plan. This approach is combined with a blocking procedure to take into account the different bonding of the adherents. The blocking procedure is a useful tool, typical of the DoE approach, used to avoid any influence of the experimental set up or the operator, as described in $[8,18,19]$.

Four different frequencies, two amplitudes, three adhesives and three replicates lead to a total amount of 72 experiments on the bonded configuration, which can be used to estimate the influence of the primary variables and the interactions. Moreover we carried out 24 additional experiments on the reference configuration, leading to a gross total of 96 experiments. 
The statistical software Design Expert was used to build the set of experimental test to be run and to randomize the order of the experiment. The software was also used to post process the results of the analysis by means of the analysis of variance analyses (ANOVA).

\subsection{System response}

The statistical influence of the variables is evaluated in terms of two system responses. Since the shaker excitation is a sine wave at given frequency, the amplitude of the corresponding spectral component is the main output choice. In particular the outputs of the experiments are:

i. $\quad$ Spectral amplitude at excitation frequency (SA for brevity)

ii. $\quad$ Percentage of signal energy stored at excitation frequency (SE for brevity)

The SA is obtained after the FFT of the measured signal, considering the amplitude of the vibratory signal at the excitation frequency, the SE is the energy of the signal at the excitation frequency over the total energy of the system.

Since one of the level of the adhesive is a reference level obtained with a block with double mass and no adhesive, it is possible to add two other system response, by dividing the values of the experimental points by the value of the reference configuration (on average). The other two outputs are not dependent on the system configuration and allow the adhesive effect to be compared more efficiently.

iii. $\quad \mathrm{SAR}=\mathrm{SA} / \mathrm{SA}_{\text {no adhesive }} \quad$ Spectral amplitude ratio (SAR for brevity) 
iv. $\quad$ SER $=\mathrm{SE} / \mathrm{SE}_{\text {no adhesive }}$ Stored energy ratio (SAR for brevity)

\subsection{Experimental set-up}

The experimental set-up consists in a small electrodynamic shaker, a monoaxial accelerometer, an input and an output board of National Instruments. Table 2 summarizes the model and the characteristics of the components. The shaker is provided with an embedded amplifier, openchain controlled with 0-1 VRMS signal supplied by the NI myDAQ board. The shaker has three different amplitude gain which can be selected by the user, but which are kept constant for all the tests. The accelerometer is connected to the NI-9233 board which is specifically designed for IEPE devices. The aluminium blocks have a $1 \mathrm{~cm} x 1 \mathrm{~cm}$ square base and $1.2 \mathrm{~cm}$ height. The reference block has the same square base but a 2.4 height, simulating two single blocks connected together, being negligible the adhesive weight. All the blocks have been tapped in order to fix it with the shaker head and the accelerometer by means of threads. The blocks were bonded using a rig to maintain alignment between the upper and lower one and a small dead weight $(0.1 \mathrm{~kg})$ to apply a sufficient pressure to entire adhesive layer. The excess of adhesive is squeezed out and removed with a blade immediately after the applications, since two adhesives are very fast and cures in seconds. In order to ensure a proper curing of all the three adhesives we kept all the joints at room temperature for $48 \mathrm{~h}$. This procedures ensure a quite constant layer of adhesive, with an estimated nominal thickness of $0.05 \mathrm{~mm}$. The resulting setup is showed in

Figure 1a and Figure 1b. The curing procedure was carried out at room temperature for $48 \mathrm{~h}$ in order to ensure complete polymerization of all the adhesives. 
In each test the head of shaker moves harmonically with characteristics listed in Table 1 . The amplitude is not measured in absolute "g" value, but as percentage of the maximum control voltage. The sampling frequency is $50 \mathrm{kHz}$ and the acquisition time is 2 seconds. The acquisition system waits a couple of seconds before starting to avoid acquisition of transient effects of the shaker.

\section{RESULTS And DISCUSSION}

\subsection{Post Processing of the data}

Vibration data are post-processed in National Instruments' LabVIEW environment. The two seconds of acquisition are split in three contiguous parts of 0.5, 1 and 0.5 seconds respectively, and only the central part of the signal is used (1 second), in order to avoid transient effect on the acquisition. The resulting spectrum resolution $-1 \mathrm{~Hz}-$ is still sufficient to clearly match the excitation frequencies.

In order to obtain the system response, the power spectrum of the vibration data is computed as reported in (1)

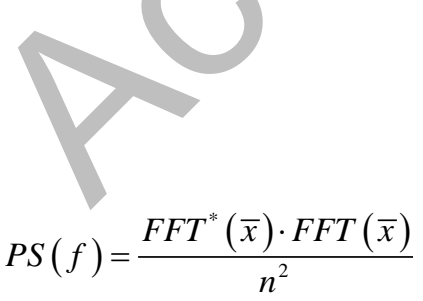


where FFT* denotes the complex conjugate of the Fast Fourier Transform (FFT) of vibration data $(\bar{X})$ and $n$ is the number of elements of samples. In an ideal condition the sine excitation signal of the shaker - measured by the accelerometer - generates a single peak in the frequency domain at the excitation frequency $f_{e x}$. On equal terms except the adhesive used, an interesting comparison among different adhesive type is made on two outputs (despite connected):

- The amplitude of the power spectrum at the excitation frequency. (SA)

- The ratio between the amplitude of the spectrum at the excitation frequency and the sum of all the power spectrum components, i.e. the total energy of the acquired vibration signal. (SE)

The choice of these parameters follows a simple consideration: SA is what is usually measured in experimental activity, i.e. it's an absolute value depending of the energy of input source, while SE returns the quality of the acquired signal independently of the energy of input source. The Parseval's theorem gives a further consideration regarding the choice of the power spectrum instead of a simple spectrum. In fact the Parseval's theorem states that the total energy contained in a time domain waveform is equal to the total energy of the waveform's Fourier Transform along the frequency domain. The equality is reported in equation (2)

$$
\int_{-\infty}^{+\infty}|x(t)|^{2} d t=\int_{-\infty}^{+\infty}|X(f)|^{2} d f
$$


It follows that the total energy of the signal can be computed as a simple sum of power spectrum components. As mentioned in paragraph 2.2 further parameters are obtained from SA and SE dividing by the corresponding values computed in the case of the reference configuration (SAR and SER respectively).

\subsection{ANOVA analyses}

Multivariable problems can be approached following a statistical method. The Design of Experiment procedure, a powerful statistical technique based on the analysis of variance analyses can be conveniently applied to these classes of problems. ANOVA calculates the variance (standard deviation) of a response considering a specific variable and the global variance of the responses. The ratio between these two variances is called the F-Test value.

In a stochastic (random) process F-value equals one, which means that the considered variable has no effect on the response, because it cannot be distinguished from experimental noise (or numerical error). Conversely the larger the F-Test value the more the variable influences the process. There are a number of approaches to represent the results graphically to demonstrate the effects of the variables on the system outputs. One of the most popular is the normal plot, used to estimate whether a certain set of data follows a Gaussian distribution or not. If the data approximates a straight line the phenomenon is statistically "normal" i.e. follows a stochastic 
law. The variables affecting the system response will then fall outside the normal distribution line, thus their effect cannot be ascribed to a stochastic process. The greater the deviation of the point from the normal line the larger the confidence interval (i.e. the probability that the variables are significant is higher). The half normal plot, used in this paper is interpreted in the same way as the normal plot but allows absolute values of the effects to be considered. The replicates of the system are important because they are used to build the error line because they are ruled by a stochastic law by definition.

\subsubsection{Half Normal plot of the responses}

Figure 2 shows the half-normal probability plots from an ANOVA according to [18] . The analysis was performed on two outputs of the problem (system response) described in Section 3.1. Figure 2a shows the SA, Figure $2 b$ the SE. The ANOVA aims at highlighting what are the effects that influences a physical phenomenon by comparing these effects of the output variable with the effect of the pure chance. This can be obtained by comparing the results coming from the several levels of the selected variables with the effect of the replicates, driven by the stochastic experimental error.

In Figure 2 the $\mathrm{X}$-axis represents the standardized effect associated with each factor considered. The greater the standardized effect, the higher the influence of the variable on the response. The Y-axis represents the half-normal probability associated with each effect. The half normal plot is simply obtained by the normal plot with absolute values of the response (no effect of the sign). Additional statistical information on the half normal plot construction can be found in [18]. 
These values represent the probability that the effect of the variable is significant. The solid line interpolating the points represents the error of the test (given by the replicates and the non influent high order interactions between the variables). The points that fall off the error line represent the factors that mainly affect each response. Thus, Figure 2 shows that $f$ and $A$ have the strongest influence both on SA and SE. The effect of the frequency is more relevant for SA (note the logarithmic transformation applied) while the interaction of amplitude and frequency is important for the SE only. As can be noticed by the labels of the axes in Figure 2 a transformation is applied to SA and SE in order to normalize the data. This linearization, called Box-Cox transformation, is strongly recommended in [19] dealing with experimental set of data by means of ANOVA technique, which performs better with linearized problems.

Thus in Figure 2a the effect of the variables are reported on the natural logarithm of the SA and on the SE raised to the power of 1.5. The half normal plots of the SAR and SER are reported in Figure 3a-b respectively. The Figure shows that dividing SA and SE by the reference configuration causes the adhesive type to be important in the system response, in terms of peak frequency (Figure 3a) but not in terms of percent energy of the signal (Figure 3b). The effect of the amplitude, relevant for the absolute measurement, obviously vanishes in Figure 3, because the signal is normalized over the reference.

\subsubsection{Variables interactions and relevance}

The effect of the variables in case of interaction is reported in Figure 4a for SA and in Figure 4b for SE. The same graphs are reported in Figure 5 for SAR (a) and for SER (b). These plots are interesting especially in case of variable interactions (Figure 4b) and in case of multiple variables 
involved in the problem (Figure 4a - Figure 5a), while in Figure 5b the experimental point collapse on a single line because only the excitation frequency affects the system response. The error bars in Figure 4 and Figure 5 are due to the standard deviation of the experiments in the three replicates for each configuration tested. Figure 4a shows that the amplitude of signal produce an increase in the SA, which is more pronounced at some specific frequencies ( $2500 \mathrm{~Hz}$ and $7500 \mathrm{~Hz}$ ), while is not evident at the lowest frequency. This behaviour is probably due to the dynamics (e.g. resonance) of the shaker foundation. This is consistent with the subsequent Figure 5a (despite the different ordinate), showing that a normalization with respect to the reference condition removes the variability highlighted in Figure 4a. The adhesive influence is quite evident in Figure 5a, in which it is shown that there is an influence of the adhesive in terms of amplitude of the signal, but the shape of three curves is the same, both for very stiff adhesive like the HBM X60 and very flexible polymers like the silano-modified Terostat 937.

From the point of view of an accelerometer user this behaviour is positive, because the adhesive do not disturb the signal but it only scale the amplitude. The absence of the adhesive influence in Figure 3b confirms that there is no effect of the adhesive in the shape of the measured signal. Moreover Figure 5b shows that only the frequency slightly affects SER.

\section{conclusion}

In this paper three structural adhesives have been experimentally compared to assess their transmissivity in terms of dynamic response. The chosen adhesives are comprehends the most used in accelerometers setup for diagnostics purposes in both laboratory and on-field 
environments and other two general purpose adhesives. In particular, the first one is a two component methylmethacrylate (HBM X60), the other two are a modified silane (Terostat 737) and a cyanoacrylate (Loctite 454) adhesives and they have been used to joint two aluminium bases, one connected to an accelerometer and the other to the head of an electromagnetic shaker. A further test has been performed on a double-length aluminium base, i.e. a continuum block without any adhesive acting as reference condition. The shaker provides a sine wave vibration in terms of acceleration at different combinations of frequency and amplitude, as summarised in Table 2. A monoaxial accelerometer gives the feedback vibration signal which has been used to compute four different parameters assessing the dynamic response of the structure by hand. The output parameters are all based on the power spectrum of the vibration signal. In details they are the amplitude of the spectrum at the shaker excitation frequency (SA), the ratio between SA and the total energy of the signal (SE), and finally the values of SA and SE for a given adhesive compared to the corresponding values in the reference condition (SAR and SER respectively). A design of experiments (DOE) approach led to the salient results which are summarized in the following:

- $\quad$ Frequency and amplitude have the strongest influence both on SA and SE

- $\quad$ The effect of the frequency is more relevant for SA while the interaction of amplitude and frequency is important for the SE only

- $\quad$ SAR and SER shows the importance the adhesive type in the system response, in terms of peak frequency (SAR) but not in terms of percent energy of the signal (SER). 
- The effect of the amplitude, relevant for the absolute measurement SA and SE, obviously vanish in both SAR and SER, because the signal is normalized over the reference.

- The adhesive influence is quite evident in SAR, in which it is shown that there is an influence of the adhesive in terms of amplitude of the signal

- The variable interaction for SAR and SER parameters is the same, both for very stiff adhesive like the HBM X60 and very flexible polymers like the silano-modified Terostat 937.

Above them two results are worth mentioning as design guidelines: the response amplitude depends on the structural characteristics of the adhesive and the transfer function of the adhesive layer doesn't distort the signal regardless of the type of adhesive. Therefore, as long as the adhesive layer is thin enough the adhesive type does not influence the transmissivity of the signal and therefore it possible to use the most convenient one according to cost related evaluations or availability.

\section{Acknowledgements}

The authors wish to thank the Inter Departmental Research Center INTERMECH MoRE of the University of Modena and Reggio Emilia for the financial support and Prof. E. Dragoni and R. Rubini for the scientific support. 


\section{REFERENCES}

[1] Bowers, S.V., Piety, K.R. and Piety, R.. Sound and Vibration, 25, 14-23. (1991)

[2] Dytran Accelerometer Mounting Considerations. [accessed November 03, 2015]. http://www.dytran.com/assets/PDF/Accelerometer Mounting Considerations.pdf

[3] Introduction to Piezoelectric Accelerometers. [accessed November 03, 2015]. http://www.pcb.com/techsupport/tech_accel

[4] Metra Mess und Frequenztechnik in Radebeul. [accessed November 03, 2015]. http://www.mmf.de/pdf/an8e-accelerometer_mounting.pdf

[5] Harris, C., Piersol, A. and Paez, T. Harris' shock and vibration handbook. [accessed November 11, 2015]. (2002)

[6] Goglio, L., Peroni, L., Peroni, M. and Rossetto, M. International Journal of Adhesion and Adhesives, 28, 329-39. [accessed July 17, 2012]. (2008)

[7] O’Brien, D.J., Mather, P.T. and White, S.R. Journal of Composite Materials, 35, 883904. [accessed October 24, 2013]. (2001)

[8] Montgomery, D.C. Design and Analysis of Experiments, Wiley. John Wiley and Sons. (2004)

[9] R. Mead, S. G. Gilmour, A.M. Amazon.com: Statistical Principles for the Design of Experiments: Applications to Real Experiments. [accessed November 03, 2015]. (2012)

[10] Kwan, K.S. The Role of Penetrant Structure on the Transport and Mechanical Properties of a Thermoset Adhesive. Blacksbrurg, VirginaTech. [accessed November 03, 2015]. (1998) 
[11] ADAMS, R.D. and PEPPIATT, N.A. The Journal of Strain Analysis for Engineering Design, 9, 185-96. (1974)

[12] Gleich, D. and Tooren, M. Van. Journal of Adhesion Science and Technology, 15, 1091101. [accessed August 03, 2011]. (2001)

[13] Lucas F.M. da Silva, David A. Dillard, Bamber Blackman, R.D.A. Testing Adhesive Joints: Best Practices. John Wiley \& Sons. p. 468. [accessed November 03, 2015]. (2012)

[14] Da Silva, L.F.M. and Rodrigues, T. The Journal of Adhesion, 82, 1091-115. [accessed August 03, 2011]. (2006)

[15] Kawashita, L.F., Kinloch, A.J., Moore, D.R.Ã. and Williams, J.G. International Journal of Adhesion and Adhesives, 28, 199-210. (2008)

[16] Arenas, J.M., Narbón, J.J. and Alía, C. International Journal of Adhesion and Adhesives, 30, 160-5. [accessed October 26, 2012]. (2010)

[17] Castagnetti, D., Spaggiari, a. and Dragoni, E. The Journal of Adhesion, 87, 780-803. [accessed September 18, 2014]. (2011)

[18] Anderson, M.J. and Whitcomb, P.J. DOE Simplified: Practical Tools for Effective Experimentation, Second Edition, Productivity Press. Productivity Press; 2 edition. (2007)

[19] Mead, R. The Design of Experiments: Statistical Principles for Practical Applications. Cambridge University Press. [accessed November 03, 2015]. (1990) 
Figure 1. Experimental setup (a) and detailed picture of the adhesive mounting (b)

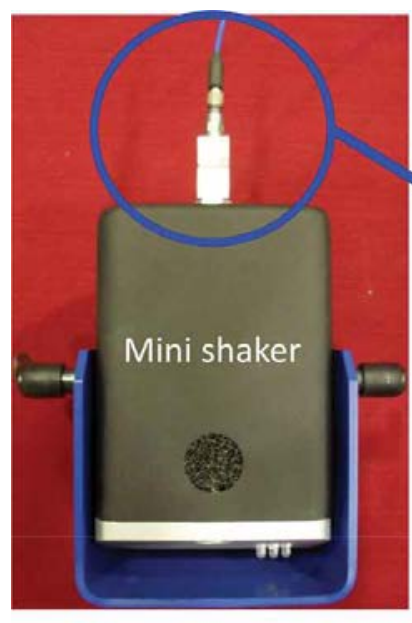

(a)

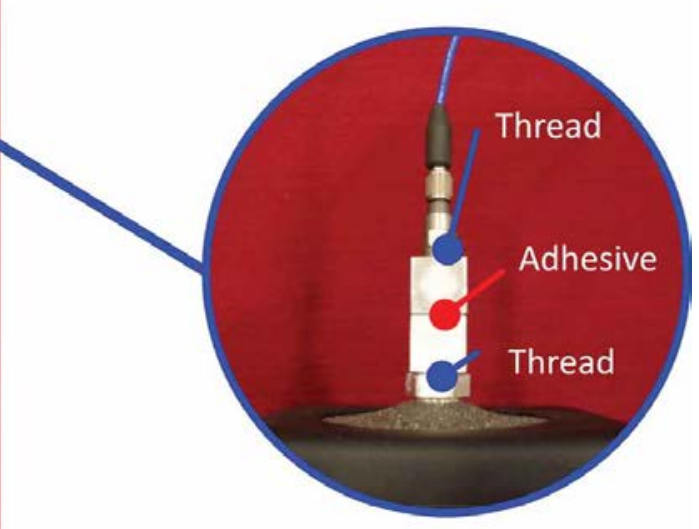

(b) 
Figure 2. Half normal plots of SA (a) and SE (b) with the proper Box-Cox transformation

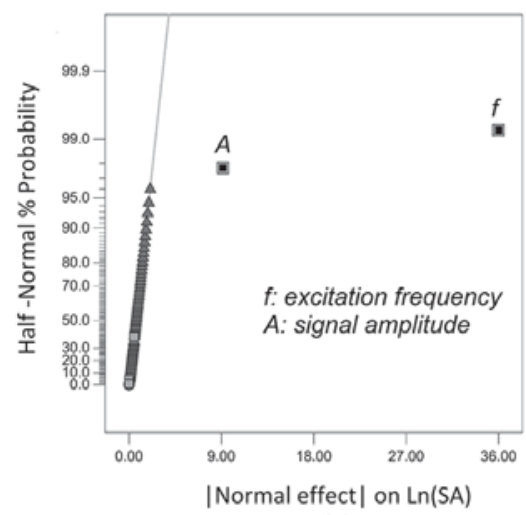

(a)

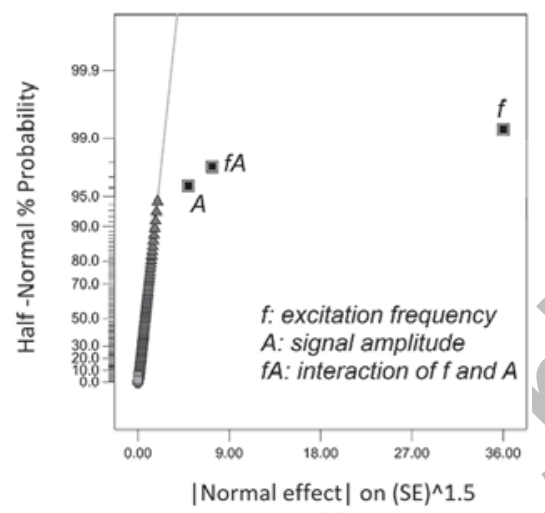

(b) 
Figure 3. Half normal plots of SAR (a) and SER (b)

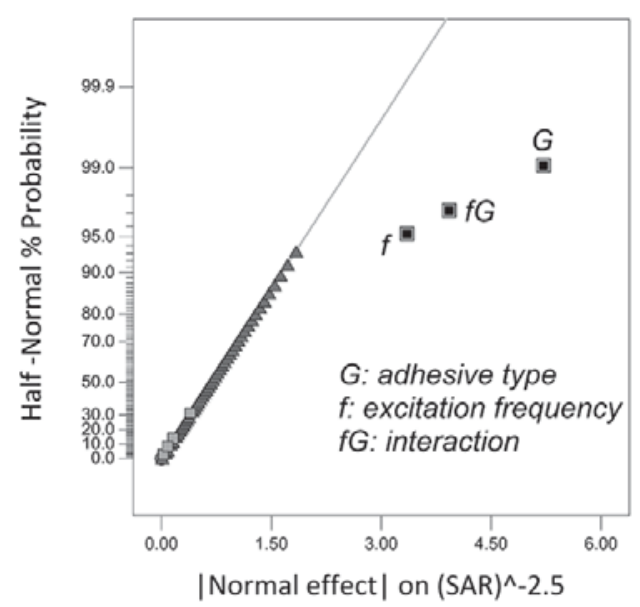

(a)

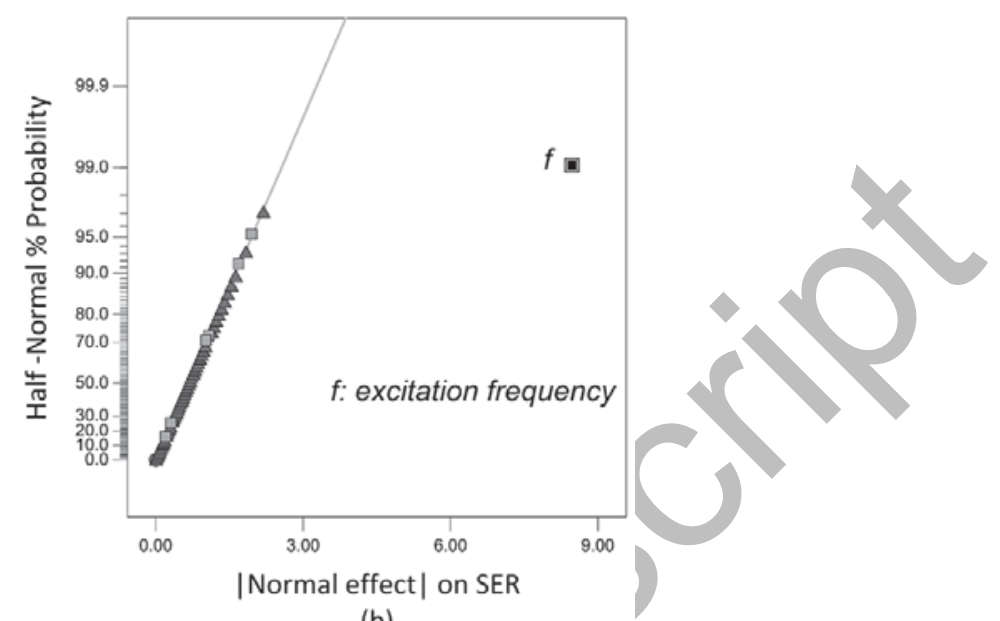

(b)

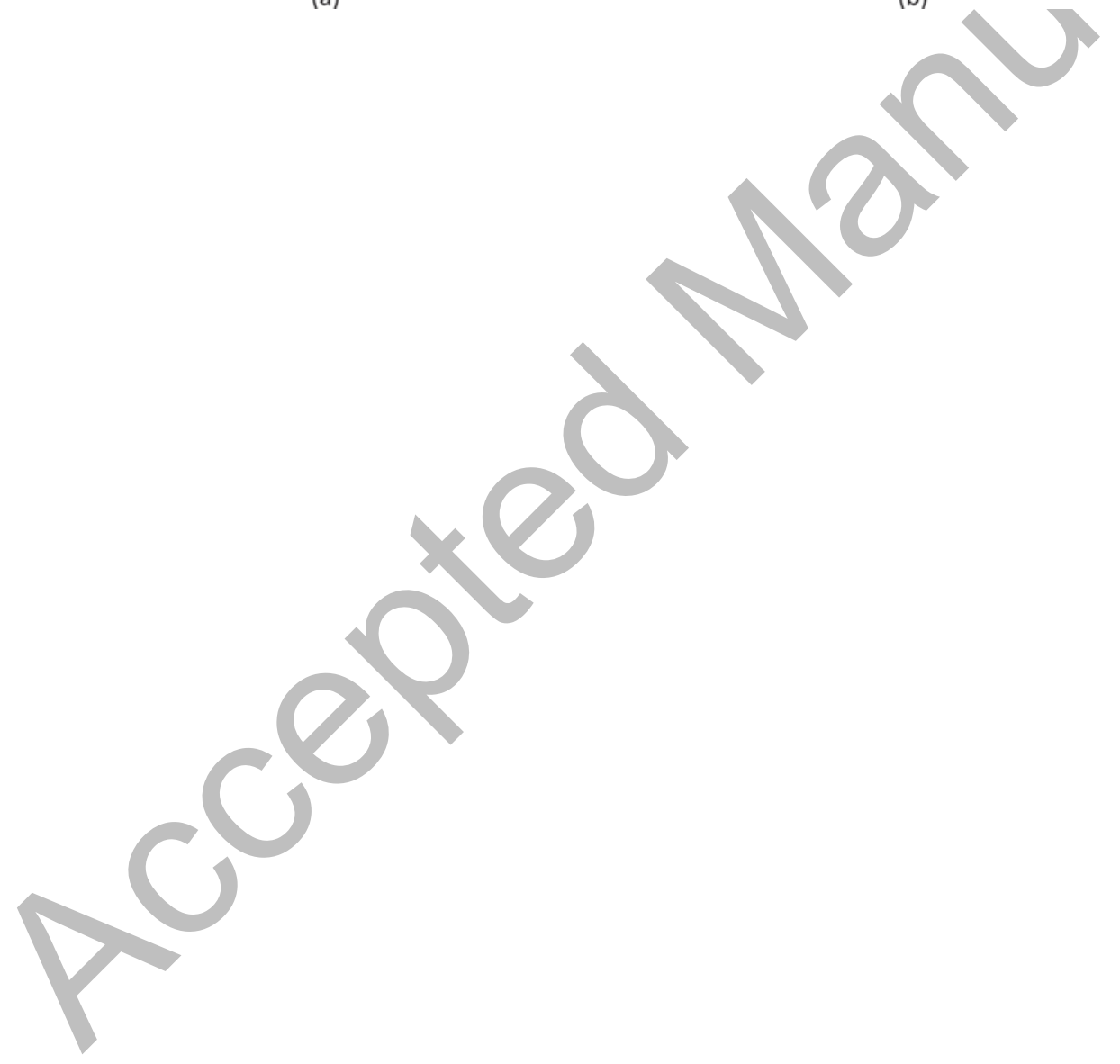


Figure 4. Variables interactions $(A, f)$ for SA (a) and SE (b) parameters

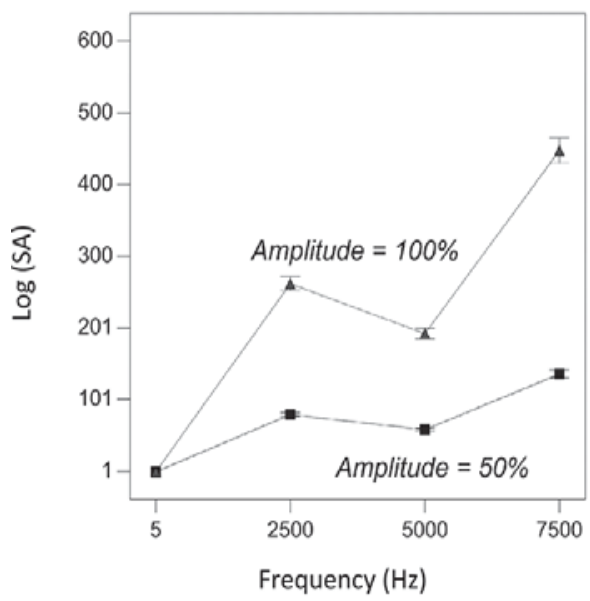

(a)

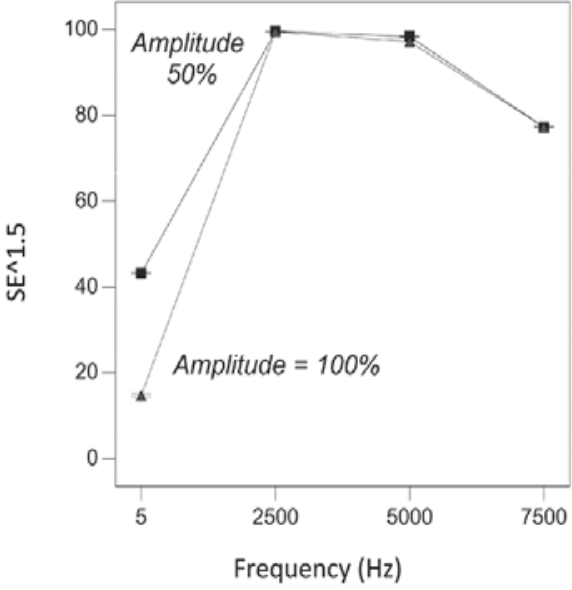

(b)

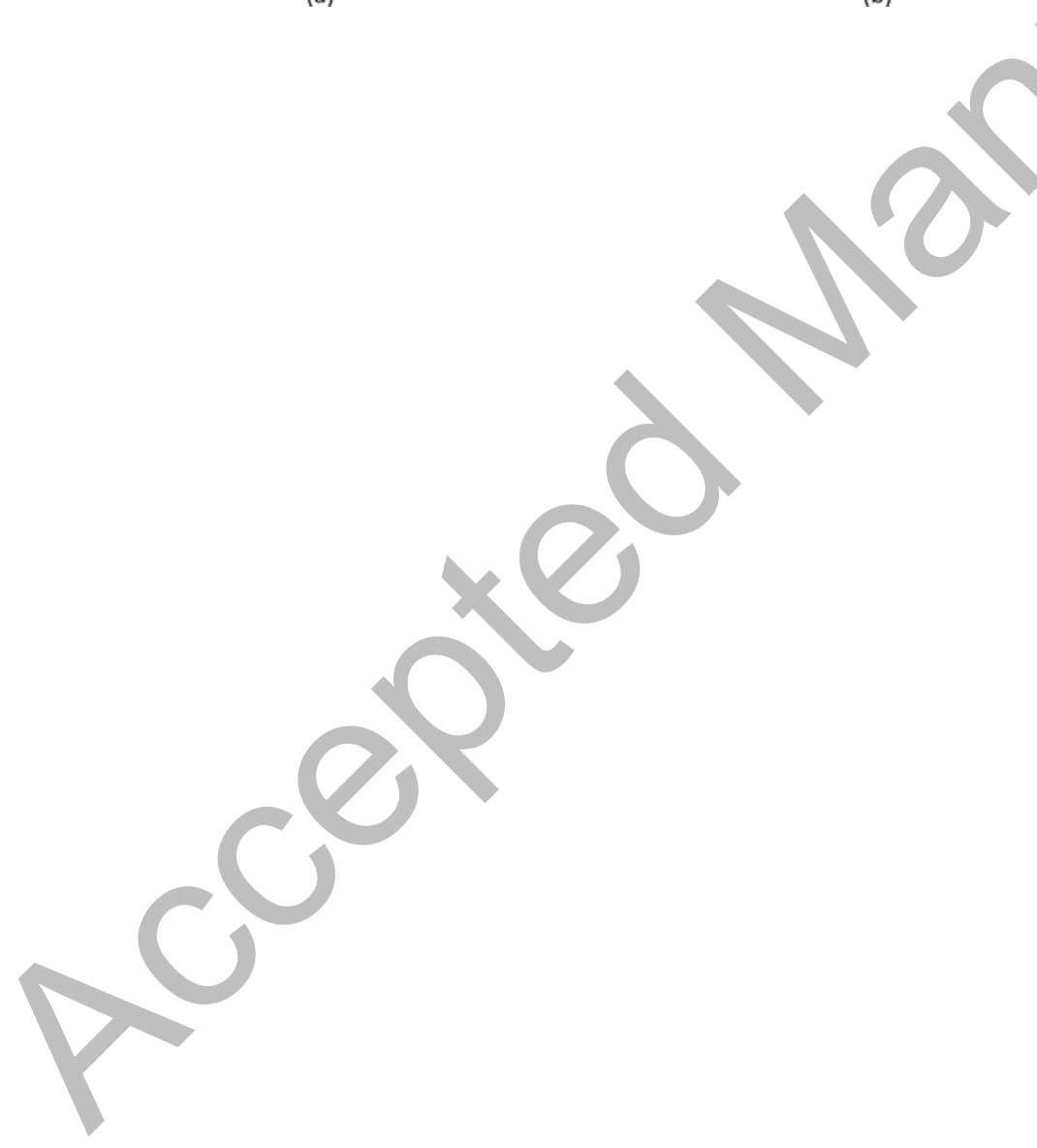


Figure 5. Variables interactions ( $f, G$ ) for SAR (a) and SER (b) parameters

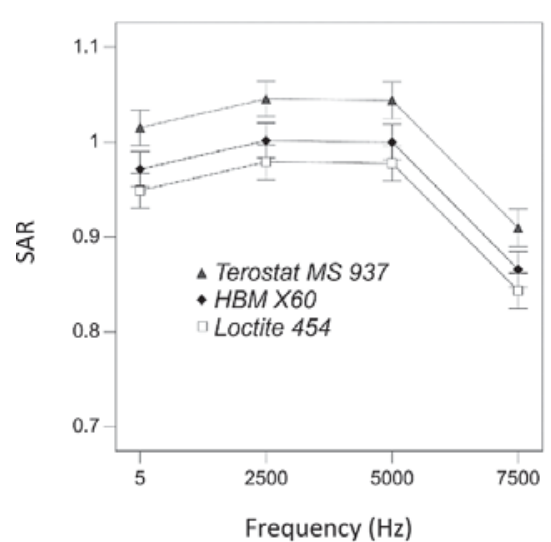

(a)

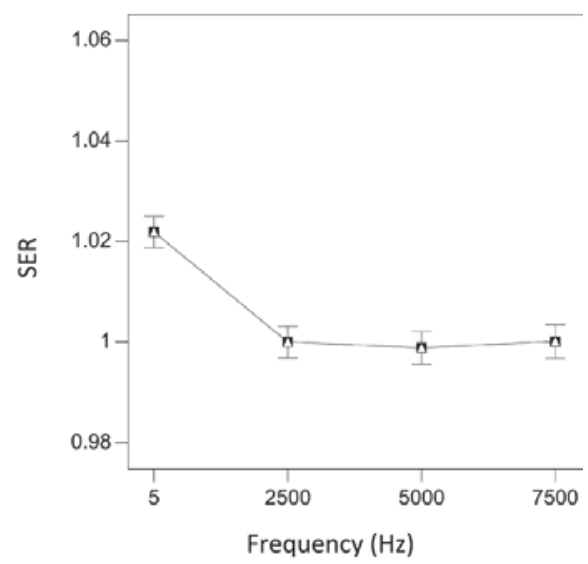

(b)

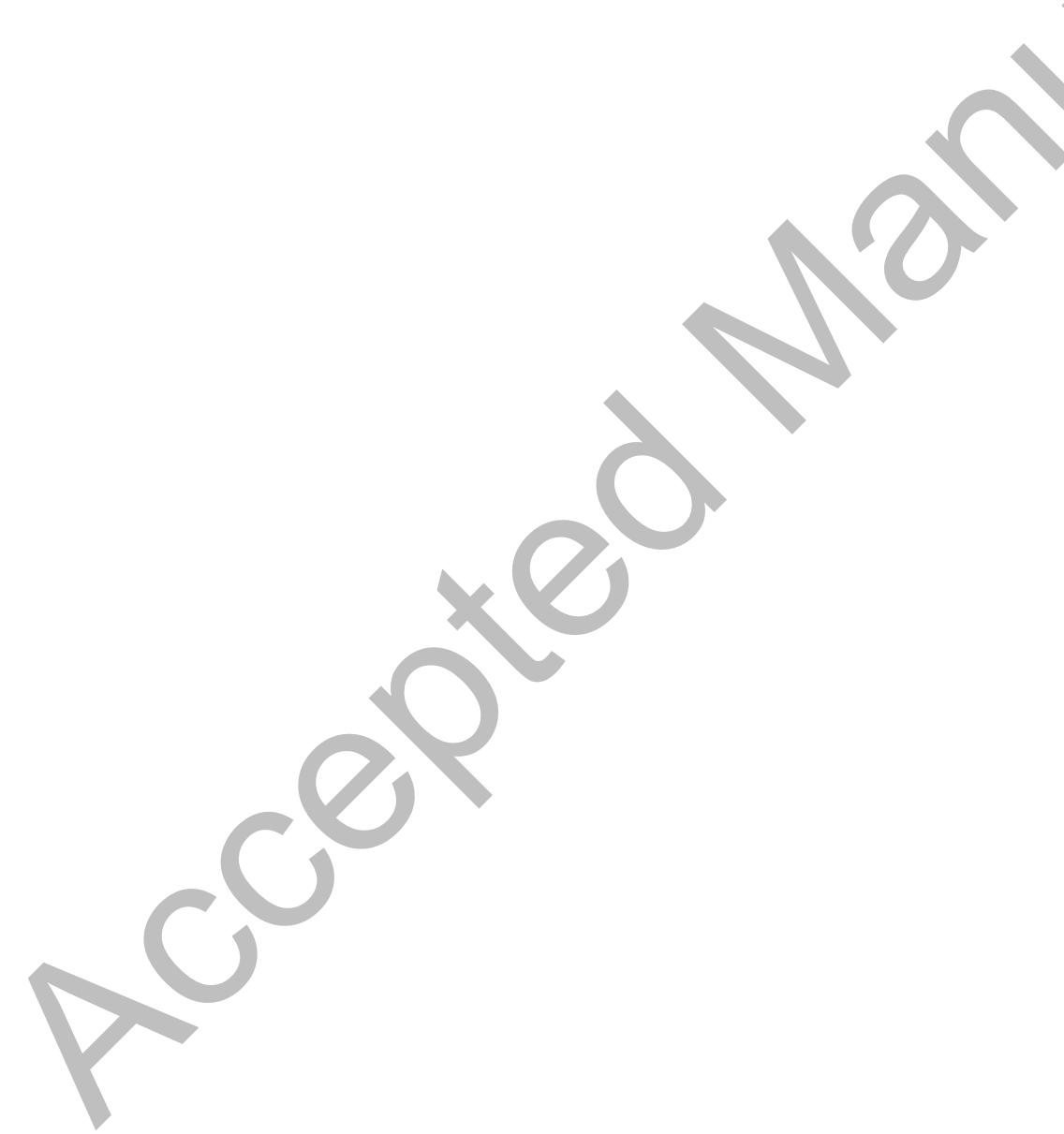


Table 1. Levels of the variables considered in the problem

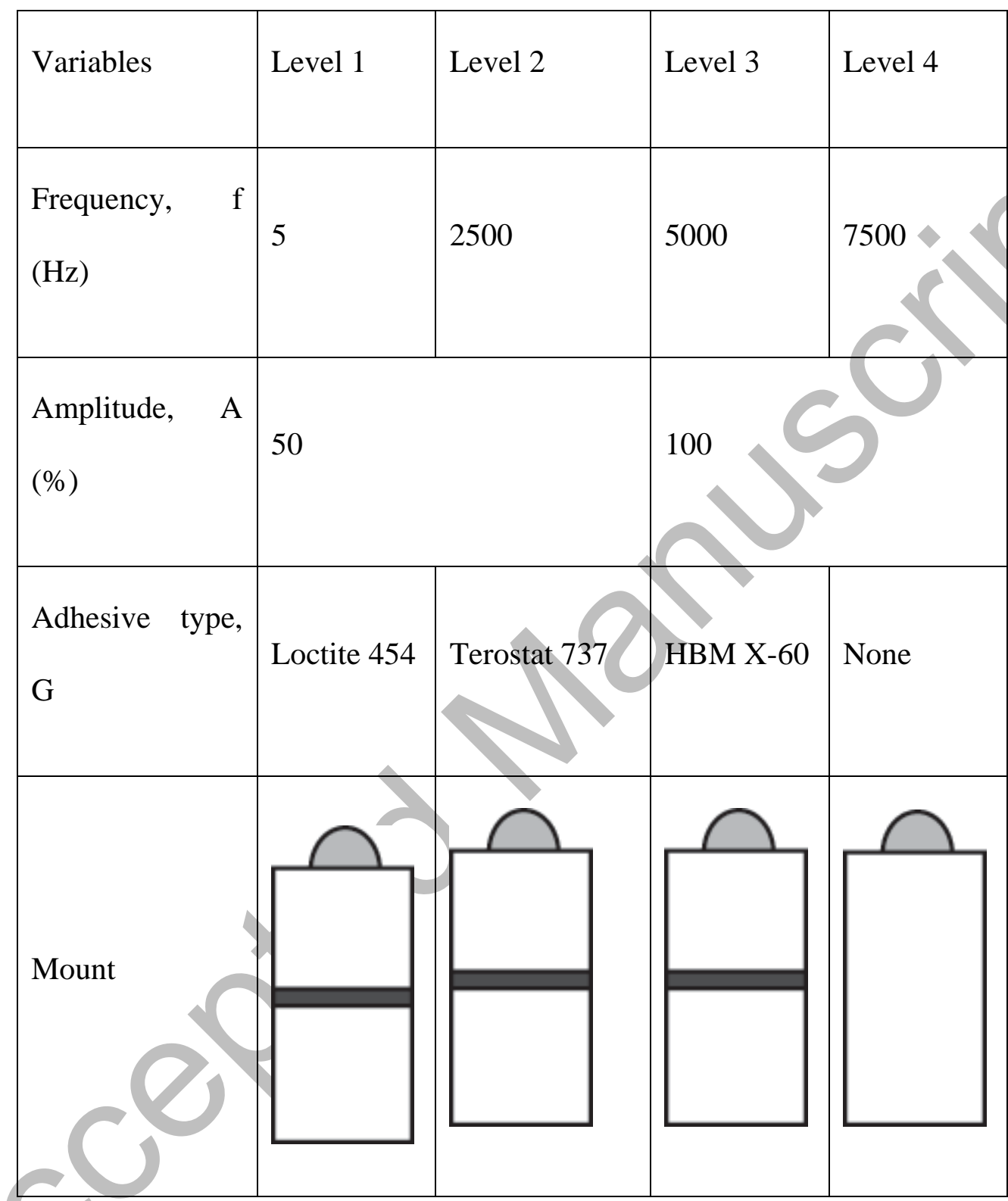


Table 2. Specifications of the components used in the experiment

\begin{tabular}{|l|l|l|}
\hline Component & Model & Specifications \\
\hline Shaker & $\begin{array}{l}\text { ModalShop } \\
\text { K2004E01 }\end{array}$ & $\begin{array}{l}\text { 20N peak sine force, frequency range up } \\
\text { to } 11 \mathrm{kHz}\end{array}$ \\
\hline Accelerometer & PCB353B18 & $\begin{array}{l}\text { Monoaxial, frequency range 1-10kHz and } \\
\text { sensitivity } 10 \mathrm{mV} / \mathrm{g}\end{array}$ \\
\hline Output board & NI myDAQ & Analog output, 200 kS/s, 16-bit \\
\hline Input board & with & NI- \\
\hline cDAQ & $51,2 \mathrm{kS} / \mathrm{s}$ per Channel, 24-Bit IEPE \\
\hline
\end{tabular}

